Chapter 7

\title{
Steady State Modeling of Three Phase Self-Excited Induction Generator Under Unbalanced/Balanced Conditions
}

\author{
A. Alsalloum and A. I. Alolah \\ Additional information is available at the end of the chapter \\ http://dx.doi.org/10.5772/53914
}

\section{Introduction}

Due to the increased emphasis on the energy issues and problems, concentration has been focused upon developing autonomous electric power supplies to be operated in remote and rural areas where electric services is unavailable from existing or nearby grids. These types of power sources can be used even in regions supplied by network grids in the event of power interruptions. Among such types that have received a notable attention and importance is the three-phase self-excited induction generator due to its numerous advantages such as simple design, robustness, and low installation and maintenance costs [1-4]. Experimental works and computer simulations have been extensively performed in order to model and analyze both steady state and transient performance of the SEIG under balanced operating conditions. However, the unbalanced operation of the SEIG has been given little attention despite its practical needs. There are two main methods to predict the steady state performance of the SEIG under balanced operating conditions. The first method is based on the generalized machine theory [5]. The second method is based on the analysis of the generalized per-phase equivalent circuit of the induction machine by applying either the loop impedance or the nodal admittance concept [6,7]. Furthermore, other studies have concentrated only on the singlephase self-excited induction generator and its voltage regulation improvement $[8,9]$. The influence of the terminal capacitance has been investigated in $[10,12]$. The previous studies have centralized mainly on modeling and analyzing the performance of SEIG under only balanced operating conditions. The major contribution of this chapter is to model the SEIG together with its excitation and load. In our steady state study, the performance of the SEIG was determined for No-load, balanced and unbalanced load and/or excitation for different SEIG and load connections. The operating conditions were found by solving the proposed 
model iteratively. An experimental setup has been built to verify the results obtained from the theoretical model. The model is generalized to cover more connection types of SEIG and/or load. It is clear that the theoretical results are in good agreement with those reported experimentally. The effect of the machine core losses is considered by representing the core resistance as a second order polynomial in terms of $X_{m}$. Furthermore, the magnetizing reactance has been included in the negative sequence equivalent circuit as a variable. The positive and negative sequence equivalent circuits are used to model the SEIG. The final characteristic equation is reached by equating both the positive-sequence and negative-sequence voltages across the SEIG and the load.

\section{Star connected generator-star connected load without a neutral connection}

The equivalent load impedance shown in Figure 1 may be described as follows;

$$
\begin{aligned}
& Z_{a}=Z_{L_{a}} / /-j X_{C_{a}} \\
& Z_{b}=Z_{L_{b}} / /-j X_{C_{b}} \\
& Z_{c}=Z_{L_{c}} / /-j X_{C_{c}}
\end{aligned}
$$

where,

$Z_{L_{a, b, c}}=$ Load impedance at base frequency connected across phase $a, b$ and $c$, respectively.

$\mathrm{X}_{C_{a, b, c}}=$ Excitation capacitor reactance at base frequency connected across phase $a, b$ and $c$, respectively.

$Z_{a, b, c}=$ Equivalent impedance of load and excitation capacitor at base frequency connected across phase $a, b$ and $c$ respectively.

At the load side, the phase voltages are:

$$
\left[\begin{array}{c}
V_{a k} \\
V_{b k} \\
V_{c k}
\end{array}\right]=\left[\begin{array}{ccc}
Z_{a} & 0 & 0 \\
0 & Z_{b} & 0 \\
0 & 0 & Z_{c}
\end{array}\right]\left[\begin{array}{c}
I_{a} \\
I_{b} \\
I_{c}
\end{array}\right]
$$

Since the load and/or the excitation capacitors are expected to be unbalanced, it is more appropriate to describe the different quantities involved in Eq. (4) in terms of their symmetrical components. Using the symmetrical components technique, the following is found: 


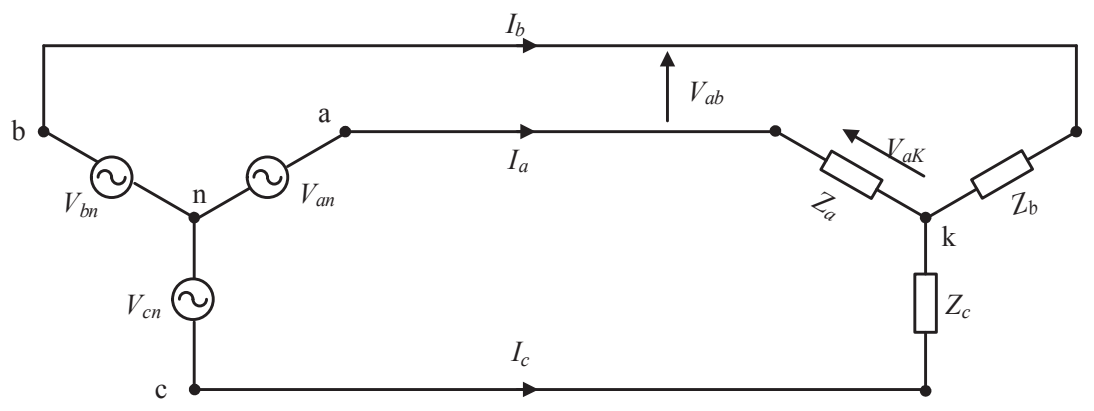

Figure 1. Star connected generator - star connected load without neutral connection.

$$
\left[\begin{array}{c}
V_{a k 0} \\
V_{a k 1} \\
V_{a k 2}
\end{array}\right]=\left[\begin{array}{lll}
Z_{0} & Z_{2} & Z_{1} \\
Z_{1} & Z_{0} & Z_{2} \\
Z_{2} & Z_{1} & Z_{0}
\end{array}\right]\left[\begin{array}{c}
I_{a 0} \\
I_{a 1} \\
I_{a 2}
\end{array}\right]
$$

Where the subscripts 0,1 , and 2 stands for zero, positive and negative sequence components, respectively. The symmetrical components of the load phase voltages may be found from the three-phase values as follows:

$$
\left[\begin{array}{c}
V_{a k 0} \\
V_{a k 1} \\
V_{a k 2}
\end{array}\right]=\left[\begin{array}{ccc}
1 & 1 & 1 \\
1 & \gamma & \gamma^{2} \\
1 & \gamma^{2} & \gamma
\end{array}\right]\left[\begin{array}{c}
V_{a k} \\
V_{b k} \\
V_{c k}
\end{array}\right]
$$

where $\gamma=1 \angle 120$

On the other hand, the three phase voltages may be found in terms of their symmetrical components by using the following transformation:

$$
\left[\begin{array}{c}
V_{a k} \\
V_{b k} \\
V_{c k}
\end{array}\right]=\left[\begin{array}{ccc}
1 & 1 & 1 \\
1 & \gamma^{2} & \gamma \\
1 & \gamma & \gamma^{2}
\end{array}\right]\left[\begin{array}{c}
V_{a k 0} \\
V_{a k 1} \\
V_{a k 2}
\end{array}\right]
$$

The transformation matrix shown in Eq. (6) can be used to find the symmetrical components of currents, namely, $I_{a 0}, I_{a 1}$, and $I_{a 2}$. The symmetrical components of the three phase impedances $Z_{a}, Z_{b}$, and $Z_{c}$ are as follows; 


$$
\left[\begin{array}{l}
Z_{0} \\
Z_{1} \\
Z_{2}
\end{array}\right]=\frac{1}{3}\left[\begin{array}{ccc}
1 & 1 & 1 \\
1 & \gamma & \gamma^{2} \\
1 & \gamma^{2} & \gamma
\end{array}\right]\left[\begin{array}{l}
Z_{a} \\
Z_{b} \\
Z_{c}
\end{array}\right]
$$

Since in an isolated-neutral star-connected load, the zero sequence component of line current (phase current) equals to zero, substituting $I_{a 0}=0$ in Eq. (5) and expanding yields:

$$
\begin{aligned}
& V_{a k 1}=Z_{0} I_{a 1}+Z_{2} I_{a 2} \\
& V_{a k 2}=Z_{1} I_{a 1}+Z_{0} I_{a 2}
\end{aligned}
$$

It can be shown using the symmetrical components technique that the relation between the positive and negative sequence components of both the line and the phase voltages are as follows:

$$
\begin{aligned}
& V_{L L 1}=\left(1-\gamma^{2}\right) V_{L N 1} \\
& V_{L L 2}=(1-\gamma) V_{L N 1}
\end{aligned}
$$

Hence,

$$
\begin{aligned}
& V_{a b 1}=\left(1-\gamma^{2}\right) V_{a k 1} \\
& V_{a b 2}=(1-\gamma) V_{a k 2}
\end{aligned}
$$

Now looking at the generator side, the following positive and negative sequence circuits of Figure 2 can be used to model the generator. As can be seen in Figure 2, the core loss resistance is taken into consideration in the positive-sequence equivalent circuit of the SEIG.

As the core loss is variable according to saturation, the core loss resistance is expressed as a function of the magnetizing reactance $\left(X_{m}\right)$ as shown in Eq. (15). Figure 3 shows the variation of the core resistance as a function of magnetizing reactance. Although this will increase the complexity of the model but the model will be closer to the actual case.

$$
\begin{aligned}
& R_{c}\left(X_{m}\right)=a_{2} X_{m}^{2}+a_{1} X_{m}+a_{0} \quad p . u . \\
& \text { where, } \\
& a_{2}=-17.159, a_{1}=33.372, a_{0}=35.699
\end{aligned}
$$




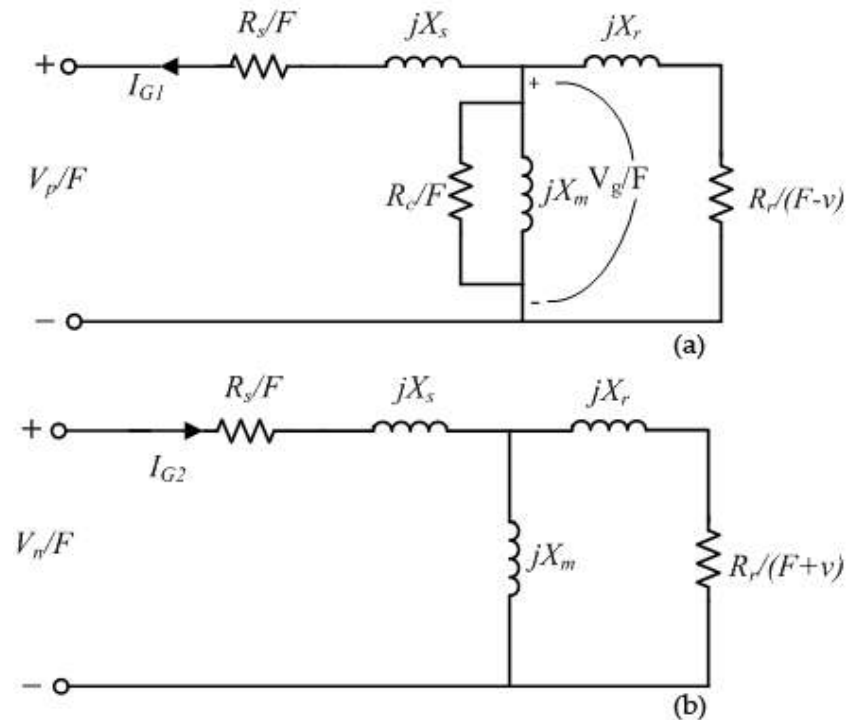

Figure 2. Induction generator equivalent circuits: (a) +ve seq. (b) -ve seq.

Furthermore, the air gap voltage may be approximated over the saturated region as a function of $X_{m}$ by the following sixth order polynomial. Figure 4 shows this variation.

$V_{g} / F=b_{6} X_{m}^{6}+b_{5} X_{m}^{5}+b_{4} X_{m}^{4}+b_{3} X_{m}^{3}+b_{2} X_{m}^{2}+b_{1} X_{m}+b_{0} \quad p . u$.

where,

$b_{6}=0.95, b_{5}=-8.28, b_{4}=28.91, b_{3}=-51.78, b_{2}=50.18, b_{1}=-25.07, b_{0}=6.21$

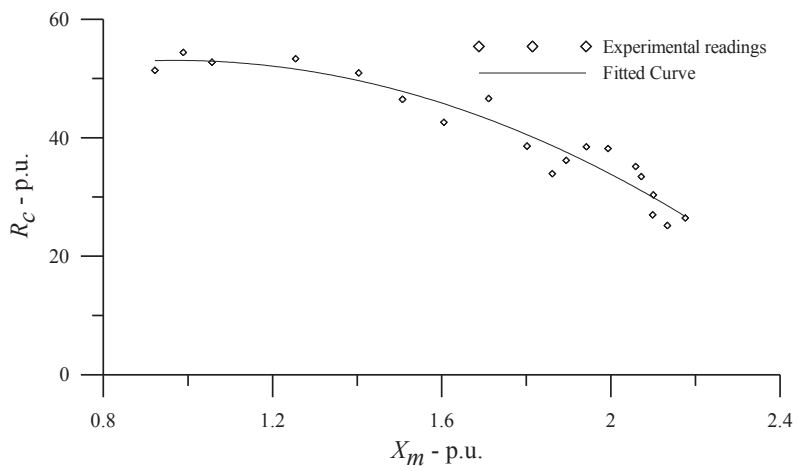

Figure 3. Variation of core resistance as a function of magnetizing reactance. 


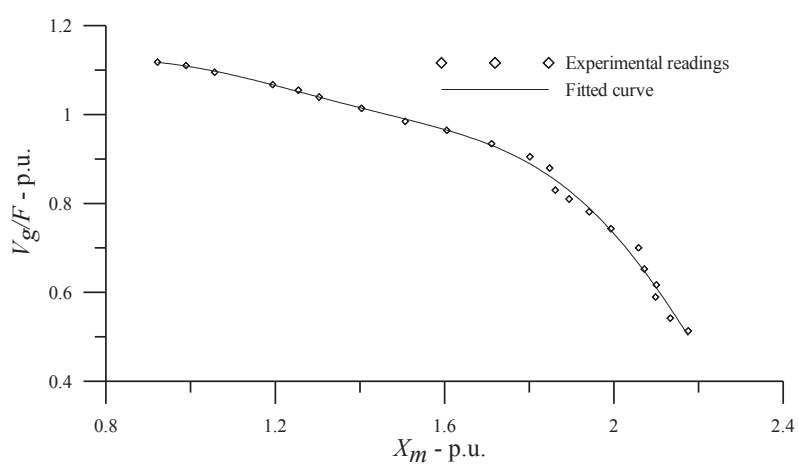

Figure 4. Variation of air gap voltage as a function of magnetizing reactance.

The terminal voltage of the positive and negative sequence equivalent circuits is given by;

$$
\begin{aligned}
& \mathrm{V}_{\mathrm{an} 1}=-\mathrm{I}_{\mathrm{G} 1} \mathrm{Z}_{\mathrm{G} 1} \\
& \mathrm{~V}_{\mathrm{an} 2}=\mathrm{I}_{\mathrm{G} 2} \mathrm{Z}_{\mathrm{G} 2}
\end{aligned}
$$

Eq. (11), Eq. (12), Eq. (17), and Eq. (18) yield the following,

$$
\begin{gathered}
V_{a b 1}=-\left(1-\gamma^{2}\right) I_{G 1} Z_{G 1} \\
V_{a b 2}=(1-\gamma) I_{G 2} Z_{G 2}
\end{gathered}
$$

where

$Z_{G 1}, Z_{G 2}$ Input impedance of positive and negative sequence equivalent circuits, respectively. Equating symmetrical components of line-to-line voltages yields:

$$
\begin{gathered}
-I_{G 1} Z_{G 1}=Z_{0} I_{a 1}+Z_{2} I_{a 2} \\
I_{G 2} Z_{G 2}=Z_{1} I_{a 1}+Z_{0} I_{a 2}
\end{gathered}
$$

Since the phase current in a star connected generator is the same as the line current, hence, 


$$
\begin{aligned}
& I_{a 1}=I_{G 1} \\
& I_{a 2}=I_{G 2}
\end{aligned}
$$

Substituting Eq. (23) and Eq. (24) into Eq. (21) and into Eq. (22) and rearranging, yields the following:

$$
\begin{aligned}
& \left(Z_{G 1}+Z_{0}\right) I_{a 1}+Z_{2} I_{a 2}=0 \\
& Z_{1} I_{a 1}+\left(Z_{0}-Z_{G 2}\right) I_{a 2}=0
\end{aligned}
$$

Solving these two equations simultaneously yields,

$$
\left(Z_{0}+Z_{G 1}\right)\left(Z_{0}-Z_{G 2}\right)-Z_{1} Z_{2}=0
$$

This is the characteristic equation of an isolated-neutral star connected induction generator. It consists of two parts, namely, the real part and the imaginary part. $I_{a 1}$ and $I_{a 2}$ does not equal zero because self-excitation is assumed to occur, hence, the real and imaginary parts of Eq. (27) must equal to zero. By substituting the machine parameters, speed, excitation capacitor values, a nonlinear equation with constant coefficients in $F$ and $X_{m}$ can be found. Solving iteratively to find the real roots of the equation that satisfies the constraints, the values of $F$ and $X_{m}$ are found; hence, the performance of the generator under these conditions can be determined. This procedure is carried out using MATHCAD® software. The flow chart describing the performance evaluation is shown in Figure 5.

\section{Star connected generator-star connected load with a neutral connection}

The connection for this case is shown in Figure 6. In this type of connection the zero sequence component of line currents is present (i.e. $I_{a 0} \neq 0$ ) while the zero sequence component of phase voltages $\left(V_{a k 0}=0\right)$ equals zero.

Expanding Eq. (5) yields:

$$
\begin{aligned}
& V_{a k 0}=Z_{0} I_{a 0}+Z_{2} I_{a 1}+Z_{1} I_{a 2} \\
& V_{a k 1}=Z_{1} I_{a 0}+Z_{0} I_{a 1}+Z_{2} I_{a 2}
\end{aligned}
$$




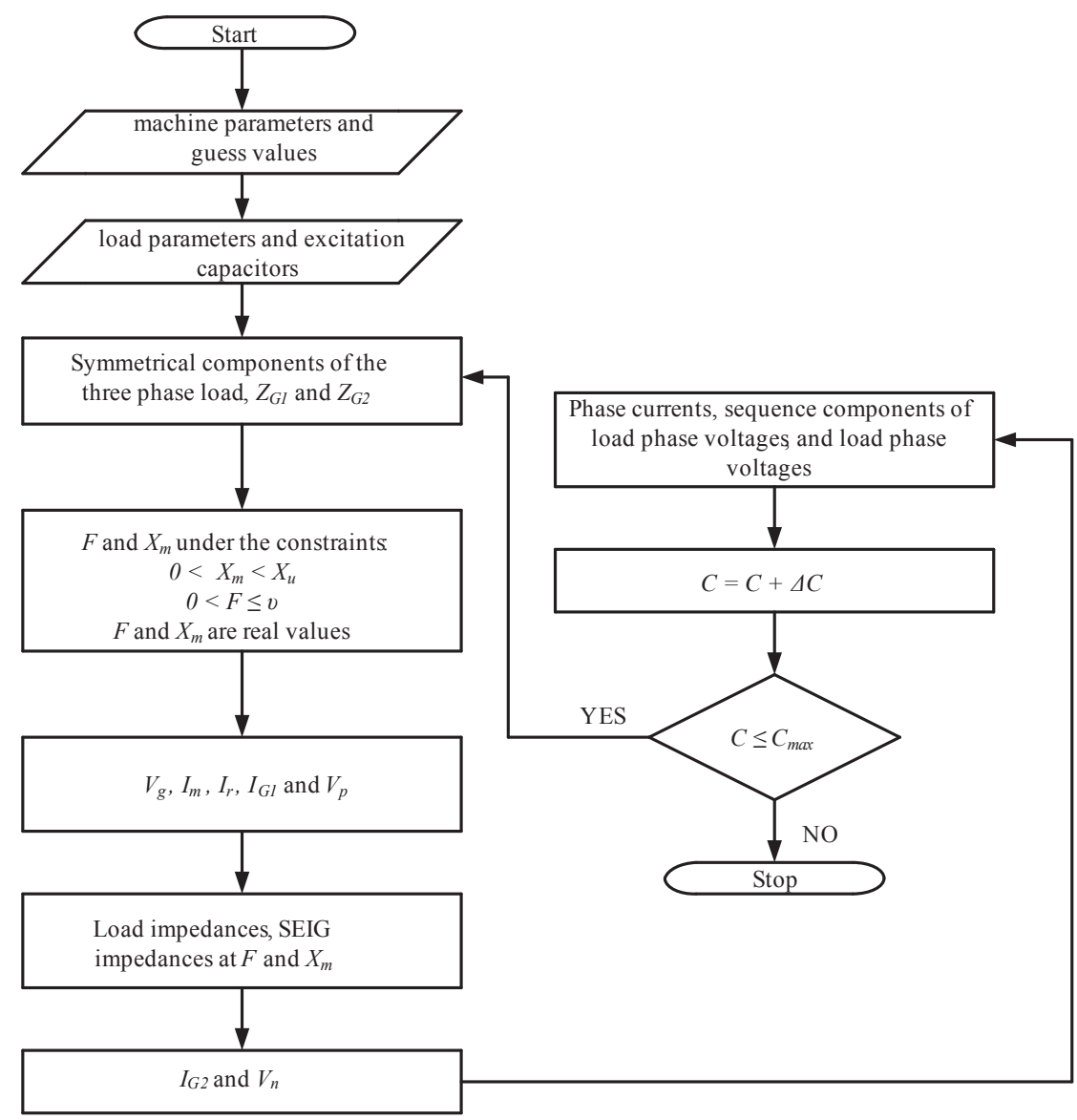

Figure 5. Flow chart describing performance evaluation.

$$
V_{a k 2}=Z_{2} I_{a 0}+Z_{1} I_{a 1}+Z_{0} I_{a 2}
$$

Substituting $V_{a k 0}=0$ in Eq. (28) and rearranging, yield:

$$
I_{a 0}=-\left(Z_{2} I_{a 1}+Z_{1} I_{a 2}\right) / Z_{0}
$$

Substituting this result in Eqs. (29) and (30), yields:

$$
V_{a k 1}=\left(Z_{0}-\frac{Z_{1} Z_{2}}{Z_{0}}\right) I_{a 1}+\left(Z_{2}-\frac{Z_{1}^{2}}{Z_{0}}\right) I_{a 2}
$$




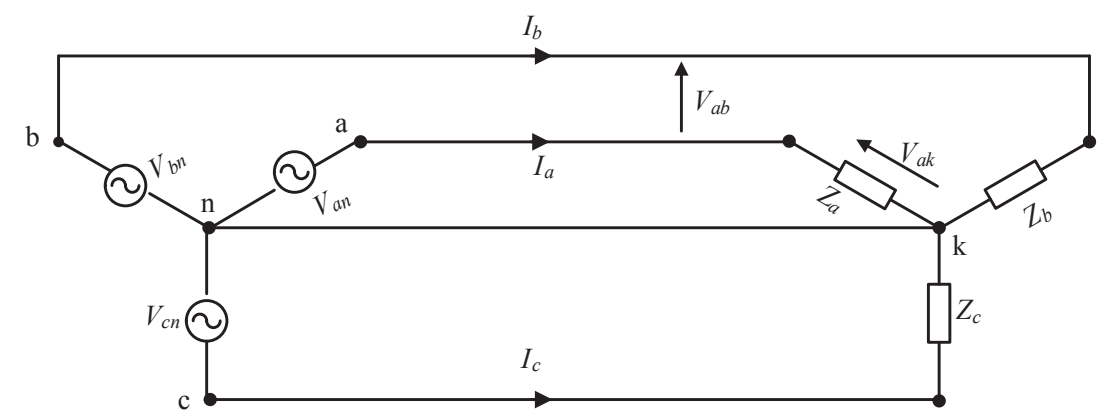

Figure 6. Star connected generator - star connected load with a neutral connection.

$$
V_{a k 2}=\left(Z_{1}-\frac{Z_{2}^{2}}{Z_{0}}\right) I_{a 1}+\left(Z_{0}-\frac{Z_{1} Z_{2}}{Z_{0}}\right) I_{a 2}
$$

Since the phase voltage of both the generator and the load are equal, hence,

$$
\begin{aligned}
& V_{a k 1}=V_{a n 1}=-I_{G 1} Z_{G 1} \\
& V_{a k 2}=V_{a n 2}=I_{G 2} Z_{G 2}
\end{aligned}
$$

Substituting Eqs. (34) and (35) into Eqs. (32) and (33), and taking into consideration that $I_{G 1}=$ $I_{a 1}$ and $I_{\mathrm{G} 2}=I_{a 2}$, yield,

$$
\begin{aligned}
& -I_{a 1} Z_{G 1}=\left(Z_{0}-\frac{Z_{1} Z_{2}}{Z_{0}}\right) I_{a 1}+\left(Z_{2}-\frac{Z_{1}^{2}}{Z_{0}}\right) I_{a 2} \\
& I_{a 2} Z_{G 2}=\left(Z_{1}-\frac{Z_{2}^{2}}{Z_{0}}\right) I_{a 1}+\left(Z_{0}-\frac{Z_{1} Z_{2}}{Z_{0}}\right) I_{a 2}
\end{aligned}
$$

Since sequence currents does not equal to zero, hence, the characteristics equation of this system equals to zero;

$$
\left(Z_{0}-\frac{Z_{1} Z_{2}}{Z_{0}}+Z_{G 1}\right)\left(Z_{0}-\frac{Z_{1} Z_{2}}{Z_{0}}-Z_{G 2}\right)-\left(Z_{1}-\frac{Z_{2}^{2}}{Z_{0}}\right)\left(Z_{2}-\frac{Z_{1}^{2}}{Z_{0}}\right)=0
$$




\section{Delta connected generator-delta connected load}

A delta-connected generator feeding a delta-connected load is shown in Figure 7, where the elements of the delta-connected load may be defined as follows,

$$
\begin{aligned}
& Z_{a b}=Z_{L_{a b}} / /-j X_{C_{a b}} \\
& Z_{b c}=Z_{L_{b c}} / /-j X_{C_{b c}} \\
& Z_{c a}=Z_{L_{c a}} / /-j X_{C_{c a}}
\end{aligned}
$$

The symmetrical components for this type of load connection are as follows:

$$
\left[\begin{array}{c}
Z_{0} \\
Z_{1} \\
Z_{2}
\end{array}\right]=\frac{1}{3}\left[\begin{array}{ccc}
1 & 1 & 1 \\
1 & \gamma & \gamma^{2} \\
1 & \gamma^{2} & \gamma
\end{array}\right]\left[\begin{array}{l}
Z_{a b} \\
Z_{b c} \\
Z_{c a}
\end{array}\right]
$$

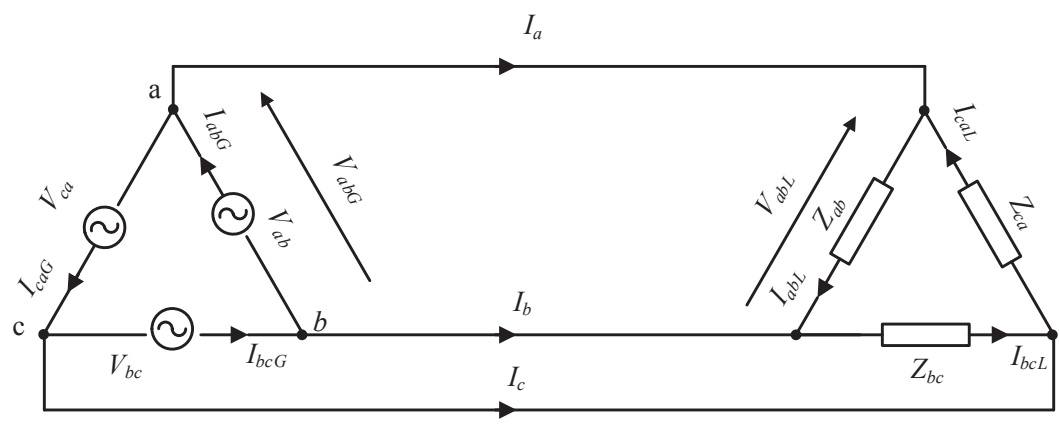

Figure 7. Delta connected generator - delta connected load.

Since the load as well as the SEIG is connected in delta, hence, the phase (line) voltage of both the generator $\left(V_{a b G}\right)$ and the load $\left(V_{a b L}\right)$ is equal. The symmetrical components of the phase voltage $\left(V_{a b}\right)$ at the load side are as follows:

$$
\left[\begin{array}{c}
V_{a b L 0} \\
V_{a b L 1} \\
V_{a b L 2}
\end{array}\right]=\left[\begin{array}{lll}
Z_{0} & Z_{2} & Z_{1} \\
Z_{1} & Z_{0} & Z_{2} \\
Z_{2} & Z_{1} & Z_{0}
\end{array}\right]\left[\begin{array}{c}
I_{a b L 0} \\
I_{a b L 1} \\
I_{a b L 2}
\end{array}\right]
$$


It is known that for a Delta connected load, $V_{a b L 0}=0$, hence, from Eq. (43)

$$
V_{a b L 0}=0=Z_{0} I_{a b L 0}+Z_{2} I_{a b L 1}+Z_{1} I_{a b L 2}
$$

This equation yields:

$$
I_{a b L 0}=-\left(Z_{2} I_{a b L 1}+Z_{1} I_{a b L 2}\right) / Z_{0}
$$

From Eq. (43)

$$
\begin{aligned}
& V_{a b L 1}=Z_{1} I_{a b L 0}+Z_{0} I_{a b L 1}+Z_{2} I_{a b L 2} \\
& V_{a b L 2}=Z_{2} I_{a b L 0}+Z_{1} I_{a b L 1}+Z_{0} I_{a b L 2}
\end{aligned}
$$

Substituting Eq. (45) in Eqs. (46) and (47), yields

$$
\begin{aligned}
& V_{a b L 1}=\left(Z_{0}-\frac{Z_{1} Z_{2}}{Z_{0}}\right) I_{a b L 1}+\left(Z_{2}-\frac{Z_{1}^{2}}{Z_{0}}\right) I_{a b L 2} \\
& V_{a b L 2}=\left(Z_{1}-\frac{Z_{2}^{2}}{Z_{0}}\right) I_{a b L 1}+\left(Z_{0}-\frac{Z_{1} Z_{2}}{Z_{0}}\right) I_{a b L 2}
\end{aligned}
$$

Since both the generator and the load are Delta connected, hence:

$$
V_{a b G 1}=V_{a b L 1} \text { and } V_{a b G 2}=V_{a b L 2}
$$

However,

$$
V_{a b G 1}=-I_{a b G 1} Z_{G 1} \text { and } V_{a b G 2}=I_{a b G 2} Z_{G 2}
$$

Substituting in Eqs. (48) and (49), yields:

$$
\begin{aligned}
& -I_{a b G 1} Z_{G 1}=\left(Z_{0}-\frac{Z_{1} Z_{2}}{Z_{0}}\right) I_{a b L 1}+\left(Z_{2}-\frac{Z_{1}^{2}}{Z_{0}}\right) I_{a b L 2} \\
& I_{a b G 2} Z_{G 2}=\left(Z_{1}-\frac{Z_{2}^{2}}{Z_{0}}\right) I_{a b L 1}+\left(Z_{0}-\frac{Z_{1} Z_{2}}{Z_{0}}\right) I_{a b L 2}
\end{aligned}
$$


It can be shown using symmetrical components technique that the sequence components of phase and line currents are related as follows:

$$
\begin{gathered}
I_{a 1}=(1-\gamma) I_{a b 1} \\
I_{a 2}=\left(1-\gamma^{2}\right) I_{a b 2}
\end{gathered}
$$

Substituting Eqs. (52) and (53) into Eqs. (50) and (51)), yields

$$
\begin{aligned}
& -\frac{I_{a 1}}{(1-\gamma)} Z_{G 1}=\left(Z_{0}-\frac{Z_{1} Z_{2}}{Z_{0}}\right) \frac{I_{a 1}}{(1-\gamma)}+\left(Z_{2}-\frac{Z_{1}^{2}}{Z_{0}}\right) \frac{I_{a 2}}{\left(1-\gamma^{2}\right)} \\
& \frac{I_{a 2}}{\left(1-\gamma^{2}\right)} Z_{G 2}=\left(Z_{1}-\frac{Z_{2}^{2}}{Z_{0}}\right) \frac{I_{a 1}}{(1-\gamma)}+\left(Z_{0}-\frac{Z_{1} Z_{2}}{Z_{0}}\right) \frac{I_{a 2}}{\left(1-\gamma^{2}\right)}
\end{aligned}
$$

Since excitation is assumed to occur and rearranging yields,

$$
\left(\frac{Z_{1} Z_{2}}{Z_{0}}-Z_{0}-Z_{G 1}\right)\left(Z_{0}-\frac{Z_{1} Z_{2}}{Z_{0}}-Z_{G 2}\right)-\left(Z_{2}-\frac{Z_{1}^{2}}{Z_{0}}\right)\left(\frac{Z_{2}^{2}}{Z_{0}}-Z_{1}\right)=0
$$

\section{Delta connected generator-star connected load}

The connection for this case is shown in Figure 8. It is known that $I_{a 0}=0$ for a star connected load, substituting in Eq. (5) and expanding $V_{a k 1}, V_{a k 2}$;

$$
\begin{aligned}
& V_{a k 1}=Z_{0} I_{a 1}+Z_{2} I_{a 2} \\
& V_{a k 2}=Z_{1} I_{a 1}+Z_{0} I_{a 2}
\end{aligned}
$$

The load sequence components of line-to-line voltage may be expressed in terms of the sequence components of line to neutral voltage as;

$$
V_{a b L 1}=\left(1-\gamma^{2}\right) V_{a k 1}
$$




$$
V_{a b L 2}=(1-\gamma) V_{a k 2}
$$

The positive and negative sequence components of generator voltage in terms of input impedances $Z_{G 1}$ and $Z_{G 2}$ are;

$$
\begin{aligned}
& V_{a b G 1}=-I_{a b G 1} Z_{G 1} \\
& V_{a b G 2}=I_{a b G 2} Z_{G 2}
\end{aligned}
$$

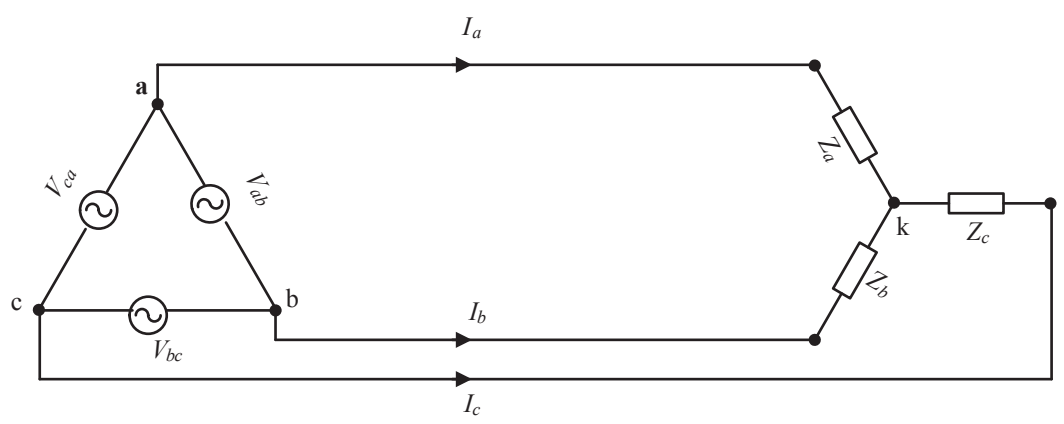

Figure 8. Delta connected generator - star connected load

These voltages equal to the load line voltages, as;

$$
\begin{aligned}
& V_{a b G 1}=V_{a b L 1} \\
& V_{a b G 2}=V_{a b L 2}
\end{aligned}
$$

Substituting Eq. (59), Eq. (60), Eq. (61), and Eq. (62) into Eq. (63) and Eq. (64), yields:

$$
\begin{aligned}
& \left(1-\gamma^{2}\right) V_{a k 1}=-I_{a b G 1} Z_{G 1} \\
& (1-\gamma) V_{a k 2}=I_{a b G 2} Z_{G 2}
\end{aligned}
$$

Substituting Eq. (57) and Eq.(58) into Eq. (65) and Eq. (66), yields:

$$
-I_{a b G 1} Z_{G 1}=\left(1-\gamma^{2}\right)\left(Z_{0} I_{a 1}+Z_{2} I_{a 2}\right)
$$




$$
I_{a b G 2} Z_{G 2}=(1-\gamma)\left(Z_{1} I_{a 1}+Z_{0} I_{a 2}\right)
$$

The symmetrical components of line current are related to the symmetrical components of phase current in a delta connected generator as follows;

$$
\begin{gathered}
I_{a 1}=(1-\gamma) I_{a b G 1} \\
I_{a 2}=\left(1-\gamma^{2}\right) I_{a b G 2}
\end{gathered}
$$

Since excitation is assumed to occur, by substituting Eq. (69) and Eq. (70) in Eqs. (67) and (68), and rearranging, yields:

$$
\left(3 Z_{0}+Z_{G 1}\right)\left(3 Z_{0}-Z_{G 2}\right)-9 Z_{1} Z_{2}=0
$$

\section{Star connected generator-delta connected load}

At the load side of Figure 9, the symmetrical components of the load are as follows:

$$
\left[\begin{array}{l}
Z_{0} \\
Z_{1} \\
Z_{2}
\end{array}\right]=\frac{1}{3}\left[\begin{array}{ccc}
1 & 1 & 1 \\
1 & \gamma & \gamma^{2} \\
1 & \gamma^{2} & \gamma
\end{array}\right]\left[\begin{array}{l}
Z_{a b} \\
Z_{b c} \\
Z_{c a}
\end{array}\right]
$$

It is known that for a delta connected load, $V_{a b L 0}=0$. Hence, from Eq. (43);

$$
V_{a b L 0}=0=Z_{0} I_{a b L 0}+Z_{2} I_{a b L 1}+Z_{1} I_{a b L 2}
$$

from this equation, it can be shown that;

$$
I_{a b L 0}=-\left(Z_{2} I_{a b L 1}+Z_{1} I_{a b L 2}\right) / Z_{0}
$$

From Eq. (43);

$$
V_{a b L 1}=Z_{1} I_{a b L 0}+Z_{0} I_{a b L 1}+Z_{2} I_{a b L 2}
$$




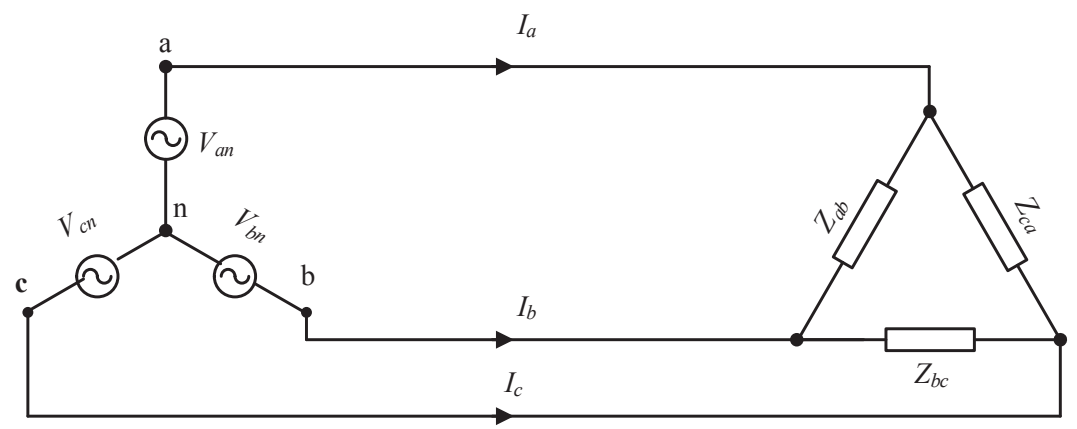

Figure 9. Star connected generator - delta connected load

$$
V_{a b L 2}=Z_{2} I_{a b L 0}+Z_{1} I_{a b L 1}+Z_{0} I_{a b L 2}
$$

Substituting Eq. (74) in Eqs. (75) and (76), yields:

$$
\begin{aligned}
& V_{a b L 1}=\left(Z_{0}-\frac{Z_{1} Z_{2}}{Z_{0}}\right) I_{a b L 1}+\left(Z_{2}-\frac{Z_{1}^{2}}{Z_{0}}\right) I_{a b L 2} \\
& V_{a b L 2}=\left(Z_{1}-\frac{Z_{2}^{2}}{Z_{0}}\right) I_{a b L 1}+\left(Z_{0}-\frac{Z_{1} Z_{2}}{Z_{0}}\right) I_{a b L 2}
\end{aligned}
$$

at the generator side,

$$
\begin{aligned}
& \mathrm{V}_{\mathrm{abG} 1}=\left(1-\gamma^{2}\right) \mathrm{V}_{\mathrm{an} 1} \\
& \mathrm{~V}_{\mathrm{abG} 2}=(1-\gamma) \mathrm{V}_{\mathrm{an} 2}
\end{aligned}
$$

It is known that

$$
\begin{aligned}
& \mathrm{V}_{\mathrm{an} 1}=-\mathrm{I}_{\mathrm{G} 1} \mathrm{Z}_{\mathrm{G} 1} \\
& \mathrm{~V}_{\mathrm{an} 2}=\mathrm{I}_{\mathrm{G} 2} \mathrm{Z}_{\mathrm{G} 2}
\end{aligned}
$$

Substituting Eq. (81) and Eq. (82) into Eq.(79) and Eq. (80), gives, 


$$
\begin{aligned}
& \mathrm{V}_{\mathrm{abG} 1}=-\left(1-\gamma^{2}\right) \mathrm{I}_{\mathrm{G} 1} \mathrm{Z}_{\mathrm{G} 1} \\
& \mathrm{~V}_{\mathrm{abG} 2}=(1-\gamma) \mathrm{I}_{\mathrm{G} 2} \mathrm{Z}_{\mathrm{G} 2}
\end{aligned}
$$

Since the line voltages at the generator and the load side are equal, hence,

$$
\begin{aligned}
& -\left(1-\gamma^{2}\right) I_{G 1} Z_{G 1}=\left(Z_{0}-\frac{Z_{1} Z_{2}}{Z_{0}}\right) I_{a b L 1}+\left(Z_{2}-\frac{Z_{1}^{2}}{Z_{0}}\right) I_{a b L 2} \\
& (1-\gamma) I_{G 2} Z_{G 2}=\left(Z_{1}-\frac{Z_{2}^{2}}{Z_{0}}\right) I_{a b L 1}+\left(Z_{0}-\frac{Z_{1} Z_{2}}{Z_{0}}\right) I_{a b L 2}
\end{aligned}
$$

It is known that,

$$
\begin{gathered}
I_{G 1}=I_{a 1} \\
I_{G 2}=I_{a 2} \\
I_{a 1}=(1-\gamma) I_{a b L 1} \\
I_{a 2}=\left(1-\gamma^{2}\right) I_{a b L 2}
\end{gathered}
$$

Since excitation is assumed to occur, by substituting Eqs. (87), (88), (89), and (90) into Eqs. (85) and (86), and rearranging yield,

$$
\left(Z_{0}+3 Z_{G 1}-\frac{Z_{1} Z_{2}}{Z_{0}}\right)\left(Z_{0}-3 Z_{G 2}-\frac{Z_{1} Z_{2}}{Z_{0}}\right)-\left(Z_{1}-\frac{Z_{2}^{2}}{Z_{0}}\right)\left(Z_{2}-\frac{Z_{1}^{2}}{Z_{0}}\right)=0
$$

\section{Generalization of steady state model}

The characteristics equations derived in the previous sections can be represented by one single general equation of the following form: 


$$
\begin{aligned}
& \left(\beta_{1} Z_{0} Z_{G 1}+\beta_{2} Z_{0}^{2}+\beta_{3} Z_{1} Z_{2}\right)\left(\beta_{4} Z_{0} Z_{G 2}+\beta_{5} Z_{0}^{2}+\beta_{6} Z_{1} Z_{2}\right) \\
& -\left(\beta_{7} Z_{0} Z_{1}+\beta_{8} Z_{2}^{2}\right)\left(\beta_{9} Z_{0} Z_{2}+\beta_{10} Z_{1}^{2}\right)=0
\end{aligned}
$$

\begin{tabular}{|c|c|c|c|c|c|c|c|c|c|c|}
\hline $\begin{array}{l}\text { Connection } \\
\text { SEIG-Load }\end{array}$ & $\beta 1$ & $\beta 2$ & $\beta 3$ & $\beta 4$ & $\beta 5$ & $\beta 6$ & $\beta 7$ & $\beta 8$ & $\beta 9$ & $\beta 10$ \\
\hline$\Delta-\Delta$ & -1 & -1 & 1 & -1 & 1 & -1 & -1 & 1 & 1 & -1 \\
\hline$\Delta-Y$ & 1 & 3 & 0 & -1 & 3 & 0 & 3 & 0 & 3 & 0 \\
\hline$Y-\Delta$ & 3 & 1 & -1 & -3 & 1 & -1 & 1 & -1 & 1 & -1 \\
\hline$Y-Y$ & 1 & 1 & 0 & -1 & 1 & 0 & 1 & 0 & 1 & 0 \\
\hline $\begin{array}{c}Y-Y \\
\text { with neutral }\end{array}$ & -1 & -1 & 1 & -1 & 1 & -1 & -1 & 1 & 1 & -1 \\
\hline
\end{tabular}

where for each connection the appropriate value of $\beta$ parameters are given in table 1 below.

Table 1. Values of Parameter $\beta$ of characteristics equation

\section{Conclusions}

A mathematical model based on the sequence equivalent circuits of the SEIG and the sequence components of the three-phase load was developed to study the performance of the SEIG in the steady state condition. Core loss resistance is included in the model as a function of $X_{m}$.

Furthermore, the magnetizing reactance $X_{m}$ is taken as a variable in the negative sequence equivalent circuit. The performance of the SEIG was determined for no-load, balanced and unbalanced load and/or excitation for different SEIG and load connections. The operating conditions were found by solving the proposed model iteratively using MATHCAD ${ }^{\circledR}$ software as described in section 1. Self-excited induction generator uses excitation capacitors at the terminals. For a given speed, and load situation, there is a specific value of the excitation capacitor that ensures voltage build up. Residual magnetism is a must in SEIG to initiate excitation. In balanced mode of operation, per phase equivalent circuit is solved to find $F$ and $X_{m}$. Unbalanced operation of SEIG can be analyzed through the method of symmetrical components and the phase voltages may differ from each other notably.

An experimental setup has been built to verify the results obtained from the theoretical model. It is found that the theoretical results are in good agreement with those recorded experimentally. The model is generalized to cover all connection types of SEIG and/or load. The characteristic equation of each type may be found by substituting the appropriate parameters, i.e., $\beta_{1}$ up to $\beta_{10}$ from table 1 , in the general model.

$$
\begin{aligned}
& \left(\beta_{1} Z_{0} Z_{p}+\beta_{2} Z_{0}^{2}+\beta_{3} Z_{1} Z_{2}\right)\left(\beta_{4} Z_{0} Z_{n}+\beta_{5} Z_{0}^{2}+\beta_{6} Z_{1} Z_{2}\right) \\
& -\left(\beta_{7} Z_{0} Z_{1}+\beta_{8} Z_{2}^{2}\right)\left(\beta_{9} Z_{0} Z_{2}+\beta_{10} Z_{1}^{2}\right)=0
\end{aligned}
$$




\section{Author details}

A. Alsalloum and A. I. Alolah*

*Address all correspondence to: alolah@ksu.edu.sa

EE Depatrment, College of English, King Saud University, Riyadh, Saudi Arabia

\section{References}

[1] Murthy, S, Malik, O, \& Tandon, A. Analysis of Self-Excited Induction Generators", IEE Proc., pt. B, (129), (1982). (6), 260-265.

[2] Elder, J, Boys, J, \& Woodward, J. The Process of Self Excitation in Induction Generators", ibid, (130), pt. B., (1983). (2), 103-107.

[3] Quazene, I, \& Mcpherson, G. Analysis of Self-Excited Induction Generators", IEEE Trans., (1983). , PAS-102, 2793-2797.

[4] Raina, G, \& Malik, O. Wind Energy Conversion Using a Self-Excited Induction Generator", ibid., Power System Apparatus, (1983). , PAS-102, 3933-3936.

[5] Grantham, C, Sutanto, D, \& Mismail, B. Steady State and Transient Analysis of SelfExcited Induction Generators", IEE Proc., pt. B, (136), (1989). (2), 61-68.

[6] Malik, N, \& Mazi, A. Capacitance Requirements for Isolated Self-Excited Induction Generators", IEEE Trans., EC-2(1), (1987). , 62-69.

[7] Al-jabri, A, \& Alolah, A. Capacitance Requirements for Isolated Self-Excited Induction Generators", IEE Proc., (137), pt. B, (1990). (3), 154-159.

[8] Singh, B. and Shilpakar, "Steady State Analysis of Single Phase Self-Excited Induction Generator", ibid., (146), (1999). (5), 421-427.

[9] Ojo, O. Performance of Self-Excited Single Phase Induction Generators with Shunt, Short Shunt and Long Shunt Excitation Connections", IEEE Trans., EC-11(3), (1996). , 477-482.

[10] Wagner, C. F, \& Evans, R. D. Symmetrical Components, McGraw-Hill, Book, (1933).

[11] Malik, N, \& Al-bahrani, A. Influence of the Terminal Capacitor on the Performance Characteristics of a Self Excited Induction Generator", IEE Proc., pt. C, (1990). , 137(2), 168-173.

[12] Al-bahrani, A, \& Malik, N. Steady State Analysis and Performance Characteristics of a Three-Phase Induction Generator Self Excited with a Single Capacitor", IEEE Trans., EC-4(4), (1990). , 725-732. 